\title{
Physical activity, obesity and mortality: does pattern of physical activity have stronger epidemiological associations?
}

\author{
Adrian E. Bauman ${ }^{1}$, Anne C. Grunseit ${ }^{{ }^{*}} \mathbb{B}$, Vegar Rangul ${ }^{2}$ and Berit L. Heitmann $3,4,5,6$
}

\begin{abstract}
Background: Most studies of physical activity (PA) epidemiology use behaviour measured at a single time-point. We examined whether 'PA patterns' (consistently low, consistently high or inconsistent PA levels over time) showed different epidemiological relationships for anthropometric and mortality outcomes, compared to single time-point measure of PA.

Methods: Data were the Danish MONICA (MONItoring Trends and Determinants in CArdiovascular Disease) study over three waves 1982-3 (time 1), 1987-8 (time 2) and 1993-4 (time 3). Associations between leisure time single timepoint PA levels at time 1 and time 3, and sport and active travel at times 1 and 2 with BMl, waist, hip circumference and mortality (death from coronary heart disease (CHD) and cardiovascular disease (CVD)) were compared to 'PA patterns' spanning multiple time points. PA pattern classified participants' PA as either 1) inactive or low PA at both time points; 2) moderate level PA at time 1 and high activity at time 3; or 3) a 'mixed PA pattern' indicating a varying levels of activity over time. Similarly, sport and active travel were also classified as indicating stable low, stable high and mixed patterns.

Results: The moderately and highly active groups for PA at times 1 and 3 had up to $1.7 \mathrm{~cm}$ lower increase in waist circumference compared with the inactive/low active group. Across 'PA patterns', 'active maintainers' had a $2.0 \mathrm{~cm}$ lower waist circumference than 'inactive/low maintainers'. Waist circumference was inversely related to sport but not active travel. CHD risk did not vary by activity levels at time 1, but was reduced significantly by $43 \%$ for high PA at time 3 (vs 'inactive' group) and among 'active maintainers' (vs 'inactive/low maintainers') by 62\%. 'Sport pattern' showed stronger reductions in mortality for cardiovascular disease and CHD deaths among sport maintainers, than the single time point measures.
\end{abstract}

Conclusions: PA patterns demonstrated a stronger association with a number of anthropometric and mortality outcomes than the single time-point measures. Operationalising PA as a sustained behavioural pattern may address some of the known under-estimation of risk for poor health in PA self-report measurements and better reflect exposure for epidemiological analysis of risk of health outcomes.

Keywords: Waist circumference, Hip circumference, Cardiovascular disease

\footnotetext{
* Correspondence: anne.grunseit@sydney.edu.au

${ }^{1}$ Prevention Research Collaboration, School of Public Health, University of Sydney, Level 6, Charles Perkins Centre, Johns Hopkins Drive, Sydney, NSW 2006, Australia

Full list of author information is available at the end of the article
} 


\section{Background}

Six decades of epidemiological studies have identified the health consequences of physical inactivity, with clear and consistent evidence for a relationship with all-cause mortality and cardiovascular disease (CVD) but more inconsistently with weight gain and fat distribution [1]. For example, studies have suggested that associations between physical activity (PA) and weight gain, overweight and obesity are weak or inconsistent, and that weight development and overweight may predict physical inactivity rather than the reverse direction [2]. On the other hand, the relationship of PA with mortality shows large and consistent effect sizes. The average meta-analytic risk reduces by 33\% for all-cause mortality for the regularly active compared to the inactive [3], with slightly smaller estimates from more recent meta-analyses [4]. For moderate intensity activities only, the relative risk reduction is 19-24\% [5]. Data are similar for CVD mortality risk reductions [6]. In summary, meta-analytic reviews suggest a $25-30 \%$ risk reduction of fatal outcomes in those who are physically active compared to the inactive.

However, a methodological limitation in this field is that most PA epidemiologic studies examine activity behaviour or measured fitness at a single point in time in relation to subsequent health outcomes or mortality. The frequency of single-point-in-time exposure measure mortality studies from an earlier (1975 and 2000) and later (2014-2016) period is quantified in Additional File 1. This sample of PA and mortality epidemiological studies indicates that $91 \%$ of those published earlier period, $93 \%$ in the later period use a single time-point PA exposure. Eight studies in each of the periods have more than one PA measurement prior to the occurrence of the outcome. As PA is a complex and changing behaviour, the epidemiologic relationships between PA and outcomes may be better characterized by assessing the pattern of the behaviour over time. Unlike behaviours such as tobacco smoking (that are reasonably stable over time), behaviours such as PA or diet may vary continuously over time [7, 8]. Tracking coefficients have been estimated for adults to fall within the low to moderate range ( 0.35 to 0.65 ) but appear to be lower over longer time intervals (for example, 0.25-0.29) [9]. If the exposure measure varies temporally to a substantial degree, then the risk estimates between PA and health outcomes may be underestimated, due to misclassification bias. Further, taking a life course perspective through multiple measures at different time points may reveal additional benefits of PA by testing the effect of consistency. Therefore, for PA it may be better to examine changes in activity, or even a longer-term patterns of PA in relation to health outcomes.

A larger number of studies have examined change in PA in relation to changes in weight or waist circumference and suggest that a change in PA may be an important determinant for preventing weight gain. For example previous research has shown lower gains in waist circumference over six years amongst those in a constant active group for leisure time PA, and for men for occupational PA [10]; lower weight and waist gain among men and especially women who increased their PA over five years compared to those with a static activity level [11]; those maintaining high levels compared with those maintaining low levels over 20 years [12]; and lower weight gain among women who increased bicycling over 16 years [13]. Of the many studies examining mortality outcomes, a few have considered change in PA or cardiorespiratory fitness and subsequent risk of death [14, 15]. However, these epidemiological studies have not examined whether a 'pattern of the behaviour of being active' over time may be more reflective of a lifetime behavioural pattern and show a stronger association with health outcomes specifically when compared to a single exposure measure. One study of walking/running did conduct both single-point and change in PA analyses, but restricted the former to only those with a consistent 'PA pattern' whilst using baseline PA as the exposure and hence no comparison between the two analyses was made [16].

This paper examines 'patterns of PA' across three waves of MONICA data in Denmark, and links this 'pattern of PA' to measures of changes in weight and body fat distribution, and to all-cause mortality, CVD and CHD mortality. The research questions are whether these 'PA patterns' over time show different epidemiological relationships for weight gain and fat distribution and with mortality and CVD/CHD outcomes, compared to single time-point measures of exposure. Specifically, we compared static baseline measures of PA and subsequent health outcomes, specifically (i) PA levels baseline in 1982/3 (time 1) and health outcomes 26 years later and (ii) PA levels at the last (time 3) data collection point (1994) and health outcomes up to 13 years later; compared with (iii) the 'PA pattern' that spanned two (time 1 and time 3 ) or all three (time 1 time 2 time 3) time points. We expected that a more proximal (to outcome) exposure and a measure of exposure pattern to have stronger predictive value than a single more distal measure.

\section{Methods}

The data source for this analysis was the Danish MONICA Study [17], an international study conducted under the auspices of the World Health Organisation (WHO) to monitor trends in and determinants of mortality from CVD. Data were collected over three waves with surveys in 1982-3 (time 1), 1987-8 (time 2) and 1993-4 (time 3). In 1982, a total 4807 people, born either 1922, 1932, 1942 or 1952 were selected from the Central Person Register as a sex and age stratified random sample of the population in the 
Western part of the Copenhagen area [18]. Subjects not born in Denmark were excluded $(n=226)$. The remaining 4581 subjects were found to be reasonably representative of the total Danish population with respect to sex, age, educational level, occupation, and housing, but persons employed in agriculture, horticulture, or fishery and self-employed and unskilled workers were slightly underrepresented. This group was invited to participate in a health examination at the Glostrup Hospital undertaken between 1982 and 1984; in total $3608(79 \%)$ participated. Five and 11 years later those still living who had participated in the first survey were re-invited to take part in the follow-up studies [19]. At the first re-examination in 1987-88, 2987 subjects (83\%) attended while at the second re-examination in 1993-94, 2656 subjects (74\%) attended. In total 3097 attended two or more health examinations. The project was approved by the Ethics Committee for Copenhagen county and is in accordance with the Helsinki II Declaration on Human Rights.

\section{Physical activity measures}

The leisure time PA question used was based on the questionnaire constructed by Saltin and Grimby [20] and asked respondents to report their habitual weekly exercise as (i) none (classified as 'inactive'), (ii) moderate PA less than $4 \mathrm{~h} /$ week (classified as 'moderately active'), (iii) moderate PA more than $4 \mathrm{~h} /$ week and (iv) strenuous, vigorous PA more than $4 \mathrm{~h}$ week (classified as 'highly active'). Although covering only one domain of PA, this measure has previously been shown to be a strong predictor for CVD and mortality and has been validated relating to maximal oxygen uptake [20]. The latter two categories were combined due to small group sizes into a 'highly active' category based on volume and intensity. These classifications ('inactive', 'moderately active' and 'highly active') were used for defining levels for the single time point measures of PA at time 1 and time 3.

Additionally, a new combined measure was constructed capturing 'PA pattern' over time and reflected the degree of maintenance of PA during the study period. The lowest category, 'inactive/low maintainers', included those who were inactive or moderately active at time 1 , and inactive at time 3 (and time 2 if they had data for this survey). 'Active maintainers' were those who had at least a moderate level of activity at time 1 and high activity at time 3 (and time 2 if they had data for this survey). The 'mixed PA pattern' included respondents whose PA did not follow either of these patterns (ie., did not show a stable 'inactive'or 'high activity' level as defined above).

In addition, two additional measures were used from questions asked about other domains of PA. Specifically, these asked about sport participation ("Do you do any kind of sport or exercise more than one hour per week?" (response categories: yes/no), and the frequency of active travel ("How long do you spend on an average workday walking and biking?" with the respondent estimating the number of minutes). Both sport participation and active travel were only asked at time 1 and time 2 . The sport question grouped subjects into those choosing to perform sport and those who did not, as this information could not be separated from the information on leisure time PA. For the current analysis sport level is described as 'none' versus 'any'. The question on active travel asked "About how much time, on a usual day, have you walked or 'cycled?" To create categories reflective of low, moderate and high in a comparatively high active-travel population [21], we classified respondents into low (0-19 $\mathrm{min} /$ day), moderate $(20-39 \mathrm{~min})$ and high $(40+$ mins/day), as other research has estimated Danes cycle to work for three hours per week on average [22]. The 'sport pattern' variable was constructed as people not doing any sport at both time 1 and time 2, and the 'sport maintainer' category were people reporting sport at both time points, and the remainder, who did sport once at either time 1 or time 2, were categorized as 'mixed'. Similarly for cycling/walking, those reporting nil or less than $20 \mathrm{~min} /$ day at both time points were categorized as 'low maintainers of active travel', those reporting $40+$ minutes/day at both time points were characterized as 'high maintainers', and the remainder were 'mixed pattern'.

\section{Health outcomes}

\section{Anthropometric measures}

Anthropometric measurements were taken by a trained nurse in accordance with WHO standards [23]. Body weight was measured to the nearest $0.1 \mathrm{~kg}$ using a SECA balance scale, with individuals dressed in light clothing or underwear. Height was measured to the nearest $0.5 \mathrm{~cm}$, with individuals wearing no shoes, feet close together, and head held in the horizontal plane [24]. Waist circumference was measured to the nearest $\mathrm{cm}$ midway between lower rib margin and the iliac crest in the horizontal plane. Hip circumference was measured to the nearest centimetre at the point yielding the maximum circumference using a tape measure. Analyses examined the relationship between the $\mathrm{PA}$ and BMI (both in BMI units and percentage change from baseline) from time 1 to time 3 . We also examined relations with waist- and hip circumference (only assessed at time 2 and time 3). Hip circumferences were recoded into those less than and those greater than $100 \mathrm{~cm}$, as this is a potential indicator of risk [25] and appears to be especially strong for women [26].

\section{Mortality}

Participants initially free from coronary heart disease (CHD), stroke, and cancer were followed up until 2007 
through personal identification numbers at the National Registers of Hospital Discharge and Death Registry. Three outcome variables were used to examine the relationship between the PA measures and mortality: allcause mortality, death caused by CVD and death from CHD defined according to International Classification of Diseases (ICD), Eighth and Tenth Revisions, ICD-8 codes 390-458 and ICD-10 codes I00-I52, and I60-I99 were used to evaluate death of CVD. For death from CHD ICD-8 codes $410-414$ and ICD-10 codes I20-I25 were used. Data on all participants could be retrieved from the registries.

Events were identified by record linkage to the Cause of Death Registry, including information regarding all deaths since January 1943, and the National Patient Registry including information regarding all hospitalizations since 1977 [27]. Events ascertainment was made by review of medical files for participants from the 1914 cohort who were included in 1974 and thus before 1977 [28]. Documentation of the validity of the diagnosis of myocardial infarction (International Classification of Diseases, Eighth Revision, code 410) in the National Patient Registry and the Cause of Death Registry has been published earlier [29].

\section{Analysis}

The analyses examining the relationship between PA and change in body mass index (BMI) and BMI percent, waist circumference (at time 3) used linear regression, with age, gender and education (as a proxy for socioeconomic status) as covariates; logistic regression models were used to assess the relationship with hip circumference, with the adjusted odds ratios reflecting the odds of being in the low risk $(>100 \mathrm{~cm})$ group at time 3 . Three models were run for each outcome variable: the first used PA measured at time 1, (PA1), the second used PA measured at time 3 (PA3), and the third assessed 'PA pattern', measured at time 1 and time 2 and/or 3. PA in all models was entered as a categorical variable with the lowest level of PA as the reference category. Each model excluded those respondents who did not have PA data at least at both time 1 and time $3(n=588)$, to ensure that the sample was consistent across the three analyses for each outcome. Identical procedures were used to examine sport at time1 and time 2, and 'sport participation pattern', and active travel (walk, cycle) at time1 and time2, and 'active travel pattern'. The analyses for waist and hip circumference were run including BMI at time 1 and BMI percent gain time 1 to time 3 as recommended in previous research [25].

For the analyses examining relationships with all-cause and CVD cause-specific mortality, Cox proportional hazards models and competing risk analyses respectively, with age at screening as the time of study entry and age at death/censoring as the exit time, were run. Age rather than time-on-study was used as the time scale as previous simulation studies have shown that the former method yields more accurate results because risk estimates are calculated on people of the same age [30]. Time at risk was calculated from time 3 for the PA analyses and time 2 for the sport and active travel analyses because, as stated above, to ensure comparable models only respondents attending these assessments were included in the analyses and therefore all included respondents would have had to have survived at least to those assessments). Respondents who had been diagnosed with CVD prior to baseline as recorded by the National Patient Registry $(n=189)$ were excluded from the mortality analyses in order to minimize reverse causality. Results are expressed in terms of hazard ratios (HR) (for all-cause mortality) and subhazard ratios (SHR) (for cause-specific mortality) of the category of interest compared with the reference category, along with $95 \%$ confidence intervals. The comparison group for deaths from all causes were those still alive at the censored time point, and for CVD and CHD deaths, those still alive along with those dying from other causes. As with the analyses of the anthropometric measures, models using the three different measures for PA were run on the same sample of respondents and used the relevant PA measure as a categorical variable.

All analyses were conducted using Stata 13.0 [31]. A threshold of 0.05 for statistical significance was used.

\section{Results}

The baseline sample collected in 1982/3 was comprised of $51.1 \%$ males, around a quarter of the sample in four roughly equal age groups (adults 30/31 years, 40/41 years, $50 / 51$ years and 60/61 years in 1983). The samples comprised 3609 adults in 1983, with 2998 in 1988 and 2555 in 1994; of these 2509 had matched data for the PA variables (at least time 1 and time 3), 2966 and 2960 were matched for the sport and walk/bike exposures (time 1 and 2 only) respectively. Almost a quarter of the sample had completed 12 or more years of education.

The data for PA at times 1 and 3, and the 'PA pattern' are shown in Table 1 . These data were from the matched sample, but did not differ on the prevalence of PA levels, weight or age/gender or education from the full baseline sample in 1982. PA in leisure time showed a slight decline in those reporting 'none' across the three time periods. The 'pattern of PA' over time indicated that less than $12 \%$ remained inactive ('inactive/low maintainers'), over two thirds showed a 'mixed pattern', and 19\% remained at least moderately active for four or more hours per week ('active maintainers'). Sport participation at time 1 and time 2 were similar, with around $32 \%$ reporting any sporting activity each week; the 'pattern' 
Table 1 Patterns of physical activity, sport, active travel and weight over time (confined to matched samples)

\begin{tabular}{|c|c|c|c|c|c|}
\hline \multirow[t]{2}{*}{ Measure } & & \multirow{2}{*}{$\begin{array}{l}1983 \\
\text { Time } 1\end{array}$} & \multirow{2}{*}{$\begin{array}{l}1988 \\
\text { Time } 2\end{array}$} & \multirow{2}{*}{$\begin{array}{l}1994 \\
\text { Time } 3\end{array}$} & \multirow{2}{*}{$\begin{array}{l}\text { Patterns of activity } \\
\text { or weight change }\end{array}$} \\
\hline \multirow{2}{*}{\multicolumn{2}{|c|}{ PA in leisure time ${ }^{a}$}} & & & & \\
\hline & & $\%$ & $\%$ & $\%$ & $\%$ \\
\hline Level 1 & None & 23.7 & 22.8 & 20.8 & \\
\hline Level 2 & Moderate $<4 \mathrm{~h}$ & 53.7 & 57.9 & 56.9 & \\
\hline Level 3 & Mod or strenuous $>4 \mathrm{~h}$ & 22.6 & 19.3 & 22.3 & \\
\hline \multirow[t]{3}{*}{ PA pattern ${ }^{a}$} & Inactive/low maintainers & & & & 11.6 \\
\hline & Mixed pattern & & & & 69.4 \\
\hline & Active maintainers & & & & 19.0 \\
\hline \multirow[t]{2}{*}{ Sport participation ${ }^{\text {b }}$} & $\%$ reporting nil & 68.3 & 67.8 & & \\
\hline & $\%$ reporting any & 31.7 & 32.2 & & \\
\hline \multirow[t]{3}{*}{ Sport participation pattern ${ }^{b}$} & No sport at time1 \& time2 & & & & 55.1 \\
\hline & Mixed sport reported once & & & & 25.9 \\
\hline & Sport both times & & & & 19.0 \\
\hline \multirow[t]{3}{*}{ Active travel Walk/bike ${ }^{c}$} & $<20 \mathrm{mins} / \mathrm{d}$ & 21.7 & 15.8 & & \\
\hline & 20-39 min/d & 26.7 & 25.2 & & \\
\hline & $40+\operatorname{mins} / d$ & 51.6 & 59.1 & & \\
\hline \multirow[t]{3}{*}{ Active travel pattern $^{c}$} & $<20$ mins both times & & & & 7.5 \\
\hline & Mixed pattern & & & & 55.2 \\
\hline & $>40$ mins both times & & & & 37.3 \\
\hline \multicolumn{2}{|l|}{$\operatorname{BMI}(\mu, s d)^{a}$} & $24.4(3.7)$ & $25.1(3.9)$ & $25.9(4.1)$ & \\
\hline \multicolumn{2}{|c|}{ BMI change time 1 to time $3 \mu(s d)^{a}$} & & & & $1.5(2.2)$ \\
\hline \multicolumn{2}{|c|}{ Percent $\mathrm{BMI}$ change time 1 to time $3 \mu(\mathrm{sd})^{\mathrm{a}}$} & & & & $6.3 \%(9.0)$ \\
\hline \multicolumn{2}{|c|}{ Mean waist circumference female/male in $\mathrm{cm}^{\mathrm{a}}$ (sd) } & - & $78.4 / 91.4(12.1)$ & $81.1 / 93.7(12.4)$ & $2.8 / 2.3(6.1)^{d}$ \\
\hline \multicolumn{2}{|c|}{ Hip circumference female/male in $\mathrm{cm}(\mathrm{sd})^{\mathrm{a}}$} & - & $98.1 / 98.5(7.3)$ & $99.3 / 99.3(8.0)$ & $1.3 / 0.80(3.9)^{d}$ \\
\hline
\end{tabular}

${ }^{a}$ Matched data based on $n=2509$ with a value for PA time 3 (2388 (95.2\%) had data at all three time points)

${ }^{\mathrm{b}}$ Matched data based on $n=2966$ with a value for sport time 1 and time 2

${ }^{c}$ Matched data based on $n=2960$ with a value for walk/bike time 1 and time 2

${ }^{\mathrm{d}}$ Change from time 2 to time 3 as not collected at time 1

of sport participation across both time periods showed a much lower prevalence, and $19.0 \%$ reporting participating in sport at both data collection periods. Active travel through walking or cycling was asked at time 1 and 2, and showed an increase in the proportion reporting $40+$ minutes of active travel between 1983 and 1988 (from 51.6 to 59.1\%). The proportion maintaining this high level of active travel at both time points was 37.3\%, with a small proportion reporting low active travel at both time points (7.5\%).

BMI, waist and hip circumference data are shown in the lower half of Table 1. There was an increase in BMI over time averaging one and a half BMI units from time 1 to time 3 . Waist circumference increased by $2.6 \mathrm{~cm}(2.8 \mathrm{~cm}$ for women and $2.3 \mathrm{~cm}$ for men) and hip circumference by just over a centimeter between time 2 and time $3(1.3 \mathrm{~cm}$ for women and $0.80 \mathrm{~cm}$ for men).

The relationship between PA and weight change is shown in Tables 2 and 3. Note that sensitivity analyses examining the effect of excluding those who did not have PA data at T2 (but did have data for T1 and T3, $n=121$ ) showed the findings below changed little if these people were removed the analyses. There was no relationship between $\mathrm{PA}$ at time 1 and subsequent change in BMI or BMI percent but the high PA group at time 3 showed a significantly lower 0.44 BMI unit and $1.6 \%$ BMI percent lower gain compared with the inactive group ( $p<0.01$ for both). Similar relationships were seen with the 'pattern of PA' where 'active maintainers' showed a 0.44 BMI unit and 1.5\% percent less BMI gain than the 'inactive/low maintainers' $(p=0.02)$.

The strongest associations among the anthropometric measures were with waist circumference measured at time 3. Waist circumference was significantly lower for the 'moderate' and 'high' PA groups at time 1 and at time 3 compared with the inactive or low PA group, up to $1.70 \mathrm{~cm}(p<0.01)$. There was an even stronger relationship (by almost 18\%) for the 'pattern of PA', where 'active maintainers' had on average $2.0 \mathrm{~cm}$ lower waist circumference than 'inactive/low maintainers' $(\mathrm{p}<0.01)$. 
Table 2 Adjusted $^{\mathrm{a}}$ BMI change, waist and hip circumference by leisure time physical activity pattern $(n=2508)$

\begin{tabular}{|c|c|c|c|c|c|}
\hline \multirow[b]{2}{*}{ PA measure } & & \multicolumn{3}{|c|}{ Beta coefficients $(95 \% \mathrm{Cl})$} & \multirow{2}{*}{$\begin{array}{l}\text { Adjusted odds ratios } \\
\text { Hip circumference } \\
>100 \mathrm{~cm} \text { at time } 3^{\mathrm{b}}\end{array}$} \\
\hline & & $\begin{array}{l}\text { BMI gain } \\
\text { time1 to time3 }\end{array}$ & $\begin{array}{l}\text { BMl gain } \% \\
\text { time } 1 \text { to time } 3\end{array}$ & Waist time $3[\mathrm{~cm}]^{\mathrm{b}}$ & \\
\hline \multirow{3}{*}{ PA time $1(1982 / 3)$} & & & & D & Rff \\
\hline & Moderately active & $-0.10(-0.31,0.12)$ & $-0.21(-1.05,0.62)$ & $-0.99(-1.48,-0.49)^{* *}$ & $1.07(0.78,1.47)$ \\
\hline & Highly active & $-0.04(-0.30,0.22)$ & $-0.14(-1.16,0.88)$ & $-1.70(-2.30,-1.10)^{* *}$ & $0.74(0.50,1.08)$ \\
\hline \multirow[t]{3}{*}{ PA time 3 (1994) } & Inactive & Ref & Ref & Ref & Ref \\
\hline & Moderately active & $-0.16(-0.38,0.07)$ & $-0.44(-1.32,0.43)$ & $-0.34(-0.86,0.17)$ & $0.97(0.70,1.35)$ \\
\hline & Highly active & $-0.44(-0.70,-0.17)^{* *}$ & $-1.59(2.63,-0.54)^{* *}$ & $-1.38(-1.99,-0.76)^{* *}$ & $0.80(0.55,1.18)$ \\
\hline \multirow[t]{3}{*}{ PA pattern } & Inactive/low maintainers & Ref & Ref & Ref & Ref \\
\hline & Mixed pattern & $-0.17(-0.44,0.10)$ & $-0.42(-1.50,0.66)$ & $-0.83(-1.46,-0.19)^{*}$ & $0.81(0.53,1.23)$ \\
\hline & Active maintainers & $-0.44(-0.76,-0.12)^{* *}$ & $-1.48(-2.76,-0.21)^{*}$ & $-2.01(-2.77,-1.26)^{* *}$ & $0.63(0.39,1.02)$ \\
\hline
\end{tabular}

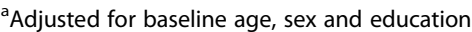

${ }^{\mathrm{b}}$ Adjusting for BMI and time 1 and BMI percent change from time 1 to time 3

${ }^{*} p \leq 0.05{ }^{* *} p \leq 0.01$

The adjusted odds ratio of an increased hip measurement at time 3 is shown in the far right hand column, and indicates levels of PA were unrelated to the odds of having a hip circumference greater than $100 \mathrm{~cm}$, irrespective of whether the exposure was single or two time-points.

Sport was not related to BMI gain or percent BMI change (Table 3) for the single or two time-point exposures. Sport at time 1 and time 2 and the patterns of 'mixed sport' and 'maintained sport' were associated with lower waist circumference at time 3 (compared to 'no sport'), with 'sport maintainers' pattern showing the strongest effect $(-1.23 \mathrm{~cm}, p<0.01) 27 \%$ larger than that for the single time point measures. 'Sport maintainers' also showed a $28 \%$ reduced odds of having a hip circumference greater than $100 \mathrm{~cm}$ at time $3(p=0.05)$ whereas the single time point measures showed no significant effect. Active travel was not related to BMI change or waist circumference for the single or two time-point exposures, and the odds of having a hip circumference $>100 \mathrm{~cm}$ were only significantly lower for active travel at time $2(20-39 \mathrm{~min}$ vs $40+\mathrm{min}, \mathrm{OR}=0.73$, $p=0.04$ ).

Table 4 shows the mortality risk for each of the PA measures, both the static time 1 and time 3 measures, the 'pattern of PA', and Table 5 shows these relationships for sport participation and active travel. All models were examined both analytically and graphically for proportional hazards and the assumption was met in all cases [32]. Risk of all-cause mortality wcat time 3 (the "highly active' group showed a $57 \%$ reduction) and the 'PA pattern' exposure, ('active maintainers' showed a 59\% reduction (HR 0.41; 95\% CI 0.28-0.59)). CVD deaths were significantly reduced in the 'highly active' group at PA at time 3 (43\% risk reduction, (HR 0.57; 95\% CI 0.350.93)) but not time 1; 'active maintainers' had a similar hazard ratio (HR 0.61; 95\% CI 0.33-1.15) but it did not reach statistically significance. The number of $\mathrm{CHD}$ (ischaemic heart disease) deaths was small $(n=69)$; only the 'active maintainers' in the 'PA pattern' exposure showed risk a reduction of $62 \%$ (HR 0.38; 95\% CI 0.15 0.96 ), and none of the single time point measures.

Table 5 shows the relationships between sport and active travel and subsequent health outcomes. 'Any sport' at time 1 or time 2 (vs 'none') lowers subsequent risk of all-cause, CVD and CHD mortality. However, larger risk reductions were noted for the 'sport pattern' of maintaining sport on both occasions (compared no sport on both occasions): $38 \%$ for all-cause (HR 0.62; $95 \% \mathrm{CI}$ 0.49-0.79), 67\% for CVD (HR 0.33; 95\% CI 0.20-0.53) and $75 \%$ for CHD (HR 0.25; 95\% CI 0.11-0.58) mortality compared to smaller protective benefits of sport at time 2 alone (34\%, 43\% and $49 \%$ risk reductions respectively). The 'mixed sport pattern' did not show a stronger effect compared to the single time-point exposures. Only the lowest active travel group at time 2, and the lowest 'pattern of active travel' categories showed significant but similar increased risk for all-cause mortality (44\% (HR $1.44 ; 95 \%$ CI 1.17-1.78) and 42\% (HR 1.42; 95\% CI $1.07-1.88)$ respectively).

\section{Discussion}

This paper proposes potential benefits of characterizing PA exposure as a 'pattern over time', especially given the variation in within-individual PA within an individual over time. The benefit was seen across a number of the examined endpoints including overall risk of premature death, risk of CVD and CHD as well as development of obesity and healthy fat patterning. There is known under-estimation of risk using PA self-report measurements [33], and a sustained behavioural pattern may better reflect true exposure. Describing 'PA patterns' rather than static and/or single point measures might reduce 





Table 4 Risk of death ${ }^{a}$ by pattern of physical activity adjusted for age, sex and education $(n=2412)$

\begin{tabular}{|c|c|c|c|c|}
\hline & & \multirow{3}{*}{$\begin{array}{l}\text { Adjusted hazard ratios }(95 \% \mathrm{Cl}) \\
\text { All-cause mortality } \\
\text { (445 deaths) }\end{array}$} & \multicolumn{2}{|c|}{ Adjusted subhazard ratios (95\% Cl) } \\
\hline & & & CVD deaths & CHD deaths \\
\hline & & & (185 deaths) & (69 deaths) \\
\hline \multirow[t]{3}{*}{ PA time1 (1982/3) } & Inactive $^{b}$ & 1.0 & 1.0 & 1.0 \\
\hline & Moderately active & $0.68(0.54-0.85)^{* *}$ & $0.89(0.62-1.28)$ & $0.88(0.48-1.62)$ \\
\hline & Highly active & $0.65(0.49-0.85)^{* *}$ & $0.73(0.47-1.15)$ & $0.83(0.40-1.72)$ \\
\hline \multirow[t]{3}{*}{ PA time 3 (1994) } & Inactive ${ }^{b}$ & 1.0 & 1.0 & 1.0 \\
\hline & Moderately active & $0.62(0.49-0.78)^{* *}$ & $0.87(0.60-1.27)$ & $0.58(0.33-1.03)$ \\
\hline & Highly active & $0.43(0.32-0.58)^{* *}$ & $0.57(0.35-0.93)^{*}$ & $0.53(0.26-1.09)$ \\
\hline \multirow[t]{3}{*}{ PA pattern } & Inactive/low maintainers ${ }^{\mathrm{b}}$ & 1.00 & 1.0 & 1.0 \\
\hline & Mixed pattern & $0.66(0.50-0.87)^{* *}$ & $1.08(0.66-1.77)$ & $0.59(0.30-1.18)$ \\
\hline & Active maintainers & $0.41(0.28-0.59)^{* *}$ & $0.61(0.33-1.15)$ & $0.38(0.15-0.96)^{*}$ \\
\hline
\end{tabular}

${ }^{a}$ Excludes those with baseline reported CVD

${ }^{\mathrm{b}}$ Reference category

${ }^{*} p \leq 0.05{ }^{* *} p \leq 0.01$

misclassification bias, and still include behaviours most proximal to the outcomes of interest, as some of the cardio-protective effects of PA may be acute [15, 34].

In this analysis of PA behaviours among Danish adults assessed over 11 years, just less than one-fifth of the cohort maintained moderate to high PA levels at both time points, a prevalence lower than at any single observation in this study. In general, irrespective of the measure, PA levels were not strongly associated with subsequent weight change. The fact that there was no relationship between baseline activity level PA (time 1) and subsequent weight gain may be due to reverse causality, as

Table 5 Risk of death ${ }^{a}$ by patterns of sport $(n=2834)$ and active travel pattern $(n=2829)$ adjusted for age, sex and education

\begin{tabular}{|c|c|c|c|c|}
\hline \multirow{2}{*}{$\overline{\text { PA measure }}$} & & \multirow{2}{*}{$\begin{array}{l}\text { Adjusted hazard ratios }(95 \% \mathrm{Cl}) \\
\text { All-cause mortality }\end{array}$} & \multicolumn{2}{|c|}{ Adjusted subhazard ratios $(95 \% \mathrm{Cl})$} \\
\hline & & & CVD deaths & CHD deaths \\
\hline & & (668 deaths) & (290 deaths) & (125 deaths) \\
\hline \multirow[t]{2}{*}{ Sport time1 $(1982 / 3)$} & None $^{c}$ & 1.0 & 1.0 & 1.0 \\
\hline & Any & $0.79(0.66,0.95)^{*}$ & $0.50(0.36,0.69)^{* *}$ & $0.44(0.26,0.74)^{* *}$ \\
\hline \multirow[t]{2}{*}{ Sport time2 (1988) } & None $^{c}$ & 1.0 & 1.0 & 1.0 \\
\hline & Any & $0.66(0.55,0.79)^{* *}$ & $0.57(0.43,0.77)^{* *}$ & $0.51(0.32,0.83)^{* *}$ \\
\hline \multirow[t]{5}{*}{ Sport pattern } & No sport at time1 \& time $2^{c}$ & 1.0 & 1.0 & 1.0 \\
\hline & Mixed sport reported once & $0.80(0.67,0.97)^{*}$ & $0.78(0.56,1.04)$ & $0.74(0.47,1.15)$ \\
\hline & Sport maintainers at time $1 \&$ time2 & $0.62(0.49,0.79)^{* *}$ & $0.33(0.20,0.53)^{* *}$ & $0.25(0.11,0.58)^{* *}$ \\
\hline & & All-cause mortality & CVD deaths & $\mathrm{CHD}$ deaths \\
\hline & & (664 deaths) & (291 deaths) & (125 deaths) \\
\hline \multirow[t]{3}{*}{ Active travel time $1^{\mathrm{b}}(1982 / 3)$} & $40+$ mins $^{c}$ & 1.0 & 1.0 & 1.0 \\
\hline & 20-39 min & $0.96(0.80,1.16)$ & $0.97(0.73,1.29)$ & $0.88(0.57,1.37)$ \\
\hline & 0-19 min & $1.11(0.92,1.34)$ & $1.13(0.85,1.50)$ & $0.75(0.47,1.19)$ \\
\hline \multirow[t]{3}{*}{ Active travel time $2^{\mathrm{b}}$ (1988) } & $40+$ mins $^{c}$ & 1.0 & 1.0 & 1.0 \\
\hline & 20-39 min & $1.09(0.89,1.32)$ & $0.98(0.72,1.32)$ & $1.06(0.67,1.66)$ \\
\hline & 0-19 min & $1.44(1.17,1.78)^{* *}$ & $1.19(0.86,1.63)$ & $1.07(0.65,1.77)$ \\
\hline \multirow[t]{3}{*}{ Active travel pattern ${ }^{\mathrm{b}}$} & $40+$ mins maintainers ${ }^{c}$ & 1.0 & 1.0 & 1.0 \\
\hline & Mixed pattern & $1.06(0.90,1.25)$ & $1.01(0.79,1.29)$ & $0.95(0.66,1.37)$ \\
\hline & 0-19 min maintainers & $1.42(1.07,1.88)^{*}$ & $1.30(0.85,1.97)$ & $1.10(0.58,2.11)$ \\
\hline
\end{tabular}

\footnotetext{
${ }^{a}$ Excludes those with baseline reported CVD

${ }^{\mathrm{b}}$ For active travel, reference category was 40+ mins, given the small numbers in the low active travel (0-19 min maintainers) category

c Reference category

${ }^{*} p \leq 0.05{ }^{* *} p \leq 0.01$
} 
earlier weight change may precede changes in PA, rather than activity being a predictor of obesity development, as also suggested by others [35]. Other Danish cohort data has shown lack of a relationship between leisure time PA and 10-year subsequent waist measurement or obesity, but did show a small effect for sports participation [36].

Nonetheless, there were significant associations with subsequent development in fat distribution, as assessed by waist circumference at time 3 , which in addition showed a dose response relationship across all measures of PA that was evident maintained even accounting for concurrent changes in weight. The strongest relationships were seen for the 'PA pattern' and waist circumference, where 'active maintainers' had two centimeters lower measures compared to the 'inactive maintainers' when adjusted for change in BMI over time. Whilst it is difficult to compare effect sizes with those from previous research because of different operationalization of PA groups, follow-up period and included covariates, the difference between those in the 'active maintainers' and 'no/low active groups' was broadly in the ranges reported elsewhere $[10,12]$. Interestingly, the finding for waist circumference was in the presence of a smaller effect on BMI, suggesting that the distribution of weight may be independently shifted towards a healthier distribution of less abdominal adiposity for those with a sustained PA and those increasing sports and active travel [37].

Previous research has repeatedly shown associations between PA and weight change when changes in activity and weight were measured concurrently, including one study which considered PA at multiple time points using repeat measures regression to demonstrate that around an hour per day of moderate PA was associated with the lowest weight gain [38]. However, this kind of repeatmeasures analysis includes regression of data from all time points, but is not the same as the 'behavioural patterning' described here. One study which did include both baseline only and PA pattern analyses appeared to show a stronger effect of PA on weight between the single-point and multiple time-point analyses, however the comparisons were between those who increased or decreased their PA from one time-point to the next compared with those who did not, irrespective of level (ie., constantly high or low) making the results difficult to compare with the current study [11]. Hence this study has quantified the effect of a particular and clinically relevant characterization of PA over time, rather than only account for within-individual change, and additionally compared it to the more frequently used single point analysis. Notably though, the more proximal single point PA measure analyses also showed strong associations with outcomes demonstrating the value of both compared with a single, distal measure.
In the present study 'PA maintainers' showed lower CHD risk than the baseline PA measure alone, although the risk reduction for all-cause mortality was similar to 'high PA' assessed at time 3 alone. Previous Danish research has shown PA protects against allcause and CVD mortality, with the latter showing relative risk reductions of $29 \%$ for moderate activity, and $44 \%$ for high PA levels [39], while a British sample of older (40-59 years) men showed risk reductions of 59\% for all-cause and 63\% for CVD-mortality for 'light/moderate active' vs. 'inactive/occasional active' maintainers [40]; the present did not show such effects in relation to CVD endpoints except for the PA at time 3. Further, the survival benefit conferred by PA of around 3-5 years [41] may also be an underestimate for the same reasons. The small number of deaths for CVD (n-185) and CHD $(n=69)$ may have contributed to the lower number of statistically significant findings despite trends mostly in the expected direction.

Sport participation measured at a single point in time in several studies, has been shown to be independently associated with mortality [42]. Our findings suggest that sport might be better characterized as a pattern, with only $15.6 \%$ participating in sport at the two time points observed. The pattern of 'maintained sport participation' was more strongly associated with $\mathrm{CHD}$ outcomes than either time 1 or time 2 sport participation in isolation. However, genetic factors, and possibly genetic pleiotropic effects, could be effect modifiers of the relationship between sport and mortality, and that should be investigated in relation to PA patterns and survival risk where such genetic information is available [43]. For active travel, which has shown protective effects in other populations $[44,45]$ there were no significant relationships with CVD or CHD mortality, only all-cause; but given the high rates of walking and cycling in and around Copenhagen [46], the unexposed group of non-active commuters was small in the current study, reducing variation in this exposure measure and making it more difficult to detect differences.

\section{Strengths and limitations}

A strength of this study is that it has proposed an arguably stronger operationalization of PA exposure for epidemiological studies, and tested it against more conventionally used measures. This study had repeat measures to characterize exposure across PA domains, including sport and active travel. The MONICA study also has a long follow-up time allowing for robust mortality analyses. One potential limitation of this study was not to adjust weight change for dietary measures and other potential confounders and covariates. However, the research purpose of this paper was not to provide 
fully adjusted independent estimates of risk for PA, but to compare across single versus 'PA pattern' measures; it is assumed that dietary and other contributions to risk are non-differential, and would not confound 'PA patterns' notably more than single-point PA estimates. It is also the case that in an observational epidemiological setting, a third variable may explain both why some individuals are physically active and have reduced risk of death, and therefore the observed relationships are not conclusive proof of causality. Another limitation is that sport and active travel were measured at two time points only (time 1 and time 2) making comparisons with the analyses for leisure time PA more difficult, and not having a PA measure in these domains more proximal to the health outcome. Further, as the sample in the PA analysis by definition must have survived to time 3 , they may constitute a healthier group than those in the sport and active travel analyses and therefore comparisons across the measures should be made with caution. Finally, the 'mixed' pattern did not distinguish between those who increased or decreased their activity from the first to second time point perhaps obscuring these effects. Further, including moderate maintainers in the mixed pattern could have masked effects of a more internally consistent mixed pattern of active travel. However, the sample sizes, especially in the cause-specific mortality analyses, were too low to further divide this group.

\section{Conclusion}

This paper presents a new hypothesis, that the concept of long term behavioural 'patterning of PA' that may extend usual epidemiological practice (as shown in Additional file 1), where most studies have assessed PA exposure at only one time point, followed by subsequent health outcomes. We found 'PA pattern' has similar effect sizes for all-cause and CVD mortality as a singlebut-proximal measure, but stronger for CHD mortality and waist circumference. BMI relationships are similar for proximal and pattern exposures. Sport pattern is consistently stronger than both single time-point measures. This method may improve exposure measurement compared to just assessing static exposure to PA at a baseline assessment and may prove to be a methodological advance for population studies at the intersection between behavioural expertise and epidemiology. Longitudinal studies could conceptualize and re-examine PA behavioural patterning in more fine-grained detail, and refine the more subtle population risks of individuals adopting, maintaining and relapsing from PA over multiple data points as potentially better predictors of health outcomes [47]. Further exploration of interaction of these patterns with concurrent sedentary behaviour time and concurrent weight status could improve the evidence base for the life course benefits of PA on health.

\section{Additional file}

Additional file 1: Bibliometric analysis of publications: what is the rate of single PA exposure measures in epidemiological studies? Bibliometric analysis of publications 1975-2000 and 2014-2016. (DOCX 15 kb)

\section{Abbreviations}

BMI: Body Mass index; CHD: Coronary Heart Disease; Cl: Confidence interval; CVD: Cardiovascular Disease; HR: Hazard ratio; ICD: International Classification of Diseases; MONICA: MONItoring Trends and Determinants in

CArdiovascular Disease; PA: Physical activity; sd: Standard deviation;

SHR: Subhazard ratio; WHO: World Health Organisation

\section{Acknowledgements}

Not applicable.

\section{Funding}

This research did not receive any specific grant from funding agencies in the public, commercial, or not-for-profit sectors. There was no funding for this research.

\section{Availability of data and materials}

Access restrictions apply to the data underlying the findings of our study. Data cannot be made publicly available for ethical and legal reasons. Public availability may compromise participant privacy, and would not comply with Danish legislation. Access to the data may be requested by an application submitted to and subsequently approved by the MONICA study Steering Boards. Contact Berit L. Heitmann (Berit.Lilienthal.Heitmann@regionh.dk) to request access.

\section{Authors' contributions}

$A B$ conceived and designed the study, conducted the bibliometric review with VR and descriptive analyses, drafted the introduction, results and discussion. AG conducted the statistical analyses, drafted the methods and assisted with editing the manuscript. BH participated in the design of the study and helped draft the manuscript. All authors read and approved the final manuscript.

\section{Ethics approval and consent to participate}

The project was approved by the Ethics Committee for Copenhagen county, is in accordance with the Helsinki II Declaration on Human Rights and the participants gave written, informed consent.

\section{Consent for publication}

Not applicable.

\section{Competing interests}

The authors declare that they have no competing interests.

\section{Publisher's Note}

Springer Nature remains neutral with regard to jurisdictional claims in published maps and institutional affiliations.

\section{Author details}

${ }^{1}$ Prevention Research Collaboration, School of Public Health, University of Sydney, Level 6, Charles Perkins Centre, Johns Hopkins Drive, Sydney, NSW 2006, Australia. ${ }^{2}$ HUNT Research Centre, Faculty of Medicine, Department of Public health and General practice, NTNU - Norwegian University of Science and Technology, Levanger, Norway. ${ }^{3}$ The Boden Institute of Obesity, Nutrition, Exercise \& Eating Disorders, University of Sydney, Sydney, NSW 2006, Australia. ${ }^{4}$ Research Unit for Dietary Studies at the Parker Institute and Institute of Preventive Medicine, Frederiksberg and Bispebjerg Hospital, Copenhagen, Denmark. Institute of Public Health, University of Southern Denmark, Odense, Denmark. ${ }^{6}$ Copenhagen Center for Preventive Medicine, Glostrup Hospital, Copenhagen Capital Region, Denmark. 
Received: 28 June 2017 Accepted: 28 September 2017

Published online: 05 October 2017

\section{References}

1. Physical Activity Guidelines Advisory Committee. Physical activity guidelines advisory committee report, 2008. Washington: US Department of Health and Human Services; 2008.

2. Pedisic Z, Grunseit A, Ding D, Chau JY, Banks E, Stamatakis E, Jalaludin BB, Bauman AE. High sitting time or obesity: Which came first? Bidirectional association in a longitudinal study of 31,787 Australian adults. Obesity. 2014; 22:2126-30.

3. Nocon M, Hiemann T, Müller-Riemenschneider F, Thalau F, Roll S, Willich SN. Association of physical activity with all-cause and cardiovascular mortality: a systematic review and meta-analysis. Eur J Cardiovasc Prev Rehabil. 2008;15: 239-46.

4. Löllgen H, Böckenhoff A, Knapp G. Physical activity and all-cause mortality: an updated meta-analysis with different intensity categories. Int J Sports Med. 2009:30:213-24.

5. Woodcock J, Franco OH, Orsini N, Roberts I. Non-vigorous physical activity and all-cause mortality: systematic review and meta-analysis of cohort studies. Int J Epidemiol. 2011;40:121-38.

6. Li J, Siegrist J. Physical activity and risk of cardiovascular disease-a metaanalysis of prospective cohort studies. Int J Environ Res Public Health. 2012; 9:391-407.

7. Schusdziarra V, Hausmann M, Wittke C, Mittermeier J, Kellner M, Wagenpfeil S, Erdmann J. Contribution of energy density and food quantity to shortterm fluctuations of energy intake in normal weight and obese subjects. Eur J Nutr. 2010:49:37-43.

8. Xue Q-L, Bandeen-Roche K, Mielenz TJ, Seplaki CL, Szanton SL, Thorpe RJ, Kalyani RR, Chaves PH, T-TL D, Ornstein K. Patterns of 12-year change in physical activity levels in community-dwelling older women: can modest levels of physical activity help older women live longer? Am J Epidemiol. 2012:176:534-43

9. Hirvensalo $M$, Lintunen T. Life-course perspective for physical activity and sports participation. Eur Rev Aging Phys Act. 2011;8:13-22.

10. Ezekwe KA, Adegboye A, Gamborg M, Heitmann BL. Associations between initial change in physical activity level and subsequent change in regional body fat distributions. Obes Facts. 2012;6:552-60.

11. May AM, Bueno-de-Mesquita HB, Boshuizen H, Spijkerman AM, Peeters $\mathrm{PH}$, Verschuren WM. Effect of change in physical activity on body fatness over a 10-y period in the Doetinchem Cohort Study. Am J Clin Nutr. 2010;92:491-9.

12. Hankinson AL, Daviglus ML, Bouchard C, Carnethon M, Lewis CE, Schreiner PJ, Liu K, Sidney S. Maintaining a high physical activity level over 20 years and weight gain. JAMA. 2010;304:2603-10.

13. Lusk AC, Mekary RA, Feskanich D, Willett WC. Bicycle riding, walking, and weight gain in premenopausal women. Arch Intern Med. 2010;170:1050-6.

14. Schnohr $\mathrm{P}$, Scharling $H$, Jensen JS. Changes in leisure-time physical activity and risk of death: an observational study of 7,000 men and women. Am J Epidemiol. 2003;158:639-44.

15. Blair SN, Kohl HW, Barlow CE, Paffenbarger RS, Gibbons LW, Macera CA. Changes in physical fitness and all-cause mortality: a prospective study of healthy and unhealthy men. JAMA. 1995;273:1093-8.

16. Williams PT. Greater weight loss from running than walking during 6.2-yr prospective follow-up. Med Sci Sports Exerc. 2013:45:706-13.

17. Kirchoff M, Schroll M, Kirkby H, Hansen B, Sanders S, Sjol A. Screening I. Danmonica Part of the MONICA Project (Multinational Monitoring of Trends and Determinants in CVD) CVD. Epidemiol Newslett. 1983:34:32.

18. Jensen $\mathrm{KH}$, Jørgensen T. Incidence of gallstones in a Danish population. Gastroenterology. 1991;100:790-4

19. Heitmann BL, Garby L. Composition (lean and fat tissue) of weight changes in adult Danes. Am J Clin Nutr. 2002;75:840-7.

20. Saltin B, Grimby G. Physiological analysis of middle-aged and old former athletes: Comparison with still active athletes of the same ages. Circulation. 1968:38:1104-15.

21. Andersen LB, Haraldsdóttir J. Changes in CHD risk factors with age: a comparison of Danish adolescents and adults. Med Sci Sports Exerc. 1994;26:967-72
22. Andersen LB, Schnohr P, Schroll M, Hein HO. All-cause mortality associated with physical activity during leisure time, work, sports, and cycling to work. Arch Intern Med. 2000;160:1621-8.

23. World Health Organization. Measuring Obesity - Classification and Description of Anthropometric Data. In: Report on a WHO Consultation of the Epidemiology of Obesity, Warsaw, 21-23 October 1987. Copenhagen: WHO; 1989.

24. Gerdes LU, Brønnum-Hansen H, Madsen M, Borch-Johnsen K, Jørgensen T, Sjøl A, Schroll M. Trends in selected biological risk factors for cardiovascular diseases in the Danish MONICA population, 1982-1992. J Clin Epidemiol. 2000;53:427-34.

25. Heitmann BL, Lissner L. Hip Hip Hurrah! Hip size inversely related to heart disease and total mortality. Obes Rev. 2011;12:478-81.

26. Lanfer A, Mehlig K, Heitmann BL, Lissner L. Does change in hip circumference predict cardiovascular disease and overall mortality in Danish and Swedish women? Obesity. 2014;22:957-63.

27. Juel K, Helweg-Larsen K. The Danish registers of causes of death. Dan Med Bull. 1999;46:354-7.

28. Schroll M. A ten-year prospective study, 1964-1974, of cardiovascular risk factors in men and women from the Glostrup population born in 1914. Dan Med Bull. 1982:29:213.

29. Andersen TF, Madsen M, Jørgensen J, Mellemkjoer L, Olsen JH. The Danish National Hospital Register. A valuable source of data for modern health sciences. Dan Med Bull. 1999;46:263-8.

30. Thiébaut A, Bénichou J. Choice of time-scale in Cox's model analysis of epidemiologic cohort data: a simulation study. Stat Med. 2004;23:3803-20.

31. StataCorp. Stata statistical software, release 13.0. College Station: Stata Corporation; 2013.

32. Cleves MA, Gould WW, Gutierrez RG. An introduction to survival analysis using Stata. College Station: STATA Corporation; 2004.

33. Prince SA, Adamo KB, Hamel ME, Hardt J, Gorber SC, Tremblay M. A comparison of direct versus self-report measures for assessing physical activity in adults: a systematic review. Int J Behav Nutr Phys Act. 2008;5:56.

34. Sherman SE, D'Agostino RB, Silbershatz H, Kannel WB. Comparison of past versus recent physical activity in the prevention of premature death and coronary artery disease. Am Heart J. 1999:138:900-7.

35. Golubic R, Ekelund U, Wijndaele K, Luben R, Khaw K-T, Wareham NJ, Brage $\mathrm{S}$. Rate of weight gain predicts change in physical activity levels: a longitudinal analysis of the EPIC-Norfolk cohort. Int J Obes. 2013;37:404-9.

36. Berentzen T, Petersen L, Schnohr P, Sørensen TI. Physical activity in leisuretime is not associated with 10-year changes in waist circumference. Scand J Med Sci Sports. 2008;18:719-27.

37. Carmienke S, Freitag M, Pischon T, Schlattmann P, Fankhaenel T, Goebel H, Gensichen J. General and abdominal obesity parameters and their combination in relation to mortality: a systematic review and metaregression analysis. Eur J Clin Nutr. 2013;67:573-85.

38. Lee I-M, Djoussé L, Sesso HD, Wang L, Buring JE. Physical activity and weight gain prevention. JAMA. 2010;303:1173-9.

39. Schnohr $P$, Lange $P$, Scharling $H$, Jensen JS. Long-term physical activity in leisure time and mortality from coronary heart disease, stroke, respiratory diseases, and cancer. The Copenhagen City Heart Study. Eur J Cardiovasc Prev Rehabil. 2006;13:173-9.

40. Wannamethee SG, Shaper AG, Walker M. Changes in physical activity, mortality, and incidence of coronary heart disease in older men. Lancet. 1998;351:1603-8.

41. Holtermann A, Marott JL, Gyntelberg F, Søgaard K, Suadicani P, Mortensen OS, Prescott E, Schnohr P. Does the benefit on survival from leisure time physical activity depend on physical activity at work? A prospective cohort study. PLoS One. 2013;8:e54548.

42. Oja P, Kelly P, Pedisic Z, Titze S, Bauman A, Foster C, Hamer M, Hillsdon M, Stamatakis E. Associations of specific types of sports and exercise with allcause and cardiovascular-disease mortality: a cohort study of 80306 British adults. Br J Sports Med. 2016; https://doi.org/10.1136/bjsports-2016-096822.

43. Karvinen S, Waller K, Silvennoinen M, Koch LG, Britton SL, Kaprio J, Kainulainen H, Kujala UM. Physical activity in adulthood: genes and mortality. Sci Rep. 2015:15:18259.

44. Hoevenaar-Blom MP, Wendel-Vos GW, Spijkerman AM, Kromhout D, Verschuren WM. Cycling and sports, but not walking, are associated with 10-year cardiovascular disease incidence: the MORGEN Study. Eur J Cardiovasc Prev Rehabil. 2011;18:41-7.

45. Hu G, Jousilahti P, Antikainen R, Tuomilehto J. Occupational, commuting, and leisure-time physical activity in relation to cardiovascular mortality among Finnish subjects with hypertension. Am J Hypertens. 2007;20:1242-50. 
46. Bassett Jr DR, Pucher J, Buehler R, Thompson DL, Crouter SE: Walking, cycling, and obesity rates in Europe, North America, and Australia. J Phys Act Health 2008; 5:795-814.

47. Rangul V, Bauman A, Holmen TL, Midthjell K. Is physical activity maintenance from adolescence to young adulthood associated with reduced CVD risk factors, improved mental health and satisfaction with life: the HUNT Study, Norway. Int J Behav Nutr Phys Act. 2012;9:144.

Submit your next manuscript to BioMed Central and we will help you at every step:

- We accept pre-submission inquiries

- Our selector tool helps you to find the most relevant journal

- We provide round the clock customer support

- Convenient online submission

- Thorough peer review

- Inclusion in PubMed and all major indexing services

- Maximum visibility for your research

Submit your manuscript at www.biomedcentral.com/submit
Biomed Central 